\title{
Genetic reprogramming of host cells by bacterial pathogens
} Guy Tran Van Nhieu ${ }^{1 *}$ and Laurence Arbibe ${ }^{2}$

\author{
Addresses: ${ }^{1}$ Unité de Communication Intercellulaire et Infections Microbiennes, Inserm U971, Collège de France, 75005 Paris, France; \\ ${ }^{2}$ Unité de Pathogénie Microbienne Moléculaire, Inserm U786, Institut Pasteur, 75015 Paris, France \\ *Corresponding author: Guy Tran Van Nhieu (guy.tran-van-nhieu@college-de-france.fr) \\ FI000 Biology Reports 2009, I:80 (doi:I0.34I0/BI-80)
}

The electronic version of this article is the complete one and can be found at: http://FI000.com/Reports/Biology/content///80

\begin{abstract}
During the course of infection, pathogens often induce changes in gene expression in host cells and these changes can be long lasting and global or transient and of limited amplitude. Defining how, when, and why bacterial pathogens reprogram host cells represents an exciting challenge that opens up the opportunity to grasp the essence of pathogenesis and its molecular details.
\end{abstract}

\section{Introduction and context}

It has been known for decades that various viruses, bacteria, and parasites can manipulate the host cell transcriptome to downregulate the host cell inflammatory response or to promote cell proliferation. With the development of microarray technology and transcriptomic analysis, a plethora of gene expression profiling studies in various pathogen models led to the characterization of Toll-like receptor (TLR)-common and -specific responses to microbial molecular patterns in the early 2000s and of a 'core host response' independent of the cell and pathogen types corresponding to a 'global alarm' to infection [1]. These studies also pointed to the fact that some pathogens affect the host cell genetic program. The large majority of studies report examples of pathogens that downregulate innate immunity. However, many pathogens and virulence factors also affect the cell cycle or induce cell growth.

How do pathogens alter the host genetic program? The various strategies that have been described so far encompass virtually every possible means of classical gene expression manipulation. Bacteria can alter host gene expression by integration of exogenous bacterial DNA in the host genome, as observed in the case of the soil bacterium Agrobacterium tumefaciens, which induces neoplastic growth in plants [2]. Obviously, such irreversible manipulation leading to permanent changes in the host cell physiology suggests an intricate coevolution between the pathogen and the host that is expressed in the context of a slow-progressing pathology at the frontier with symbiosis. Alternatively, plant pathogens have been reported to activate an antibacterial program in plant cells by inducing the synthesis of microRNA, which interferes with auxin signaling [3].

Bacterial virulence factors can act within the cytosol of host cells by altering signaling pathways regulating the transcription of proinflammatory genes. Among the most studied, the Yersinia YopJ/YopP, a bacterial factor delivered into host cells by a type III secretion system belonging to the YopJ/YopP/AvrBsT family, has been associated with multiple functions. YopJ/P is a deubiquitinating enzyme that inhibits the activation of nuclear factor-kappa-B (NF-kB) and was also shown to have an acetyltransferase activity that prevents the phosphorylation and activation of mitogen-activated protein kinase (MAPK) (Figure 1, \#1) [4-6]. Bacterial type III effectors that translocate into the host cell nucleus may also alter transcription in various ways. The plant pathogen Xanthomonas AvrBs3 family proteins are effectors that mimic a host transcriptional activator, which upregulates Upa20, a master cell regulator controlling cell size (Figure 1, \#2) [7]. The Chlamydia trachomatis CPAF (Clamydia protease activity factor) protein is secreted into the cytoplasm of host cells and is responsible for the degradation of transcription factors required for major histocompatibility complex (MHC)-protein expression or 
Figure I. How bacterial effectors manipulate host cell genetic programs

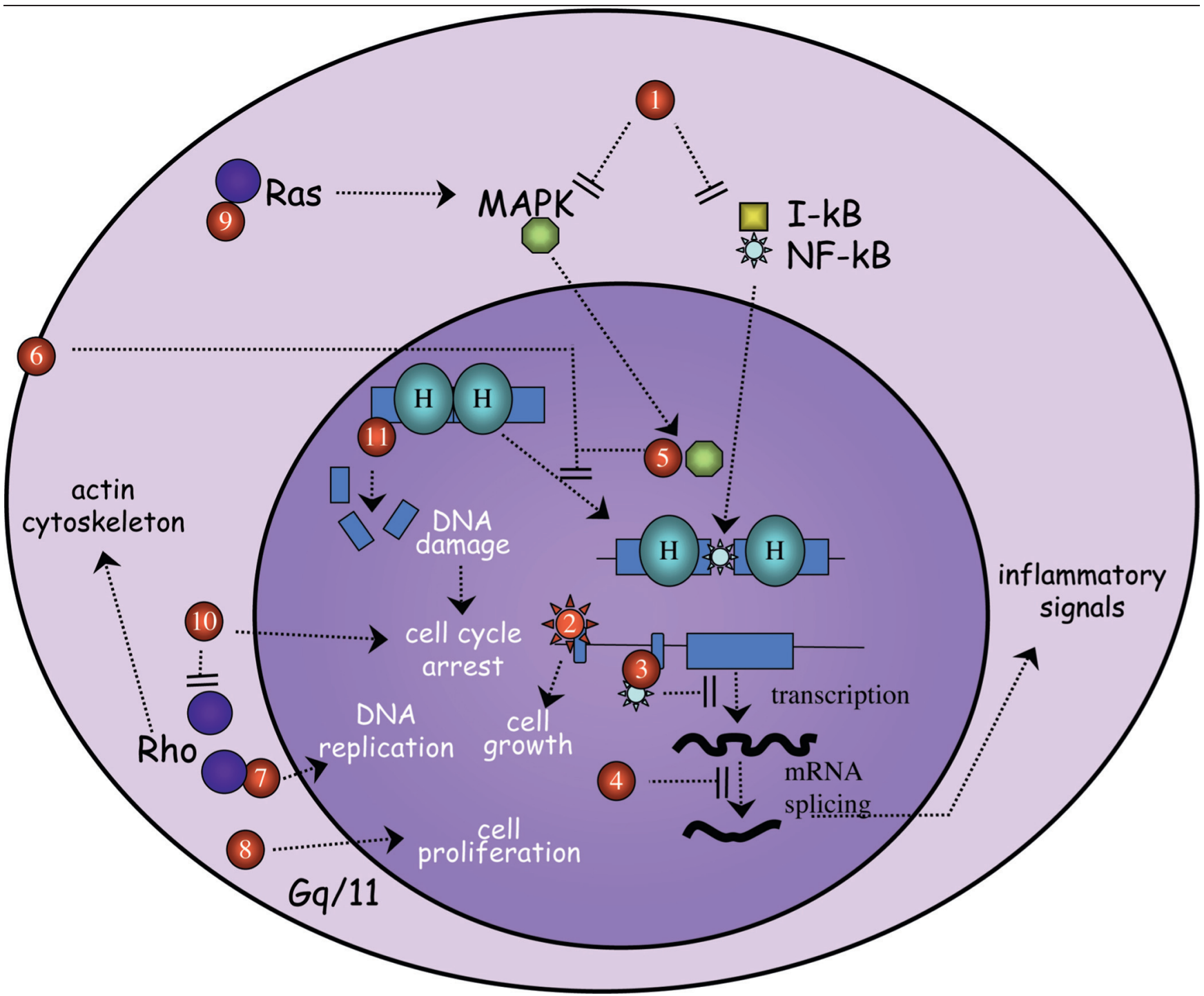

The Yersinia YopJ type III effector can affect gene expression leading to proinflammatory signals by interfering with mitogen-activated protein kinases (MAPKs) and nuclear factor-kappa-B (NF- $\kappa$ B) signaling pathways. Type III effectors that translocate in the cell nucleus can also affect the transcription of proinflammatory genes in various ways. The Xanthomonas AvrBs3 family proteins mimic a host transcriptional activator controlling cell size. Various bacterial effectors regulate the cell cycling or cell proliferation. The bacteria effectors are depicted as solid red circles with numbers referenced in the text: I, Yersinia Yopj; 2, Xanthomonas AvrBs3; 3, Xanthomonas XopD; 4, Shigella IpaH9.8; 5, Shigella OspF; 6, Listeria monocytogenes lysteriolysin O, Clostridium perfringens perfringolysin, and Streptococcus pneumoniae pneumolysin; 7, Escherichia coli cytotoxic necrotizing factors; 8, Pasteurella multocida toxin; 9, Helicobacter pylori CagA; I0, Clostridium botulinum C3 exoenzyme and E. coli cycle inhibiting factors; II, cytolethal distending toxins. H, nucleosome; I- $\kappa$ B, inhibitor of kappa-B.

the HIF1 $\alpha$ (hypoxia inducible factor $1 \alpha$ )-dependent response to hypoxia [8,9]. CPAF is also responsible for massive morphological changes and nonapoptotic death of host cells [10]. XopD, another Xanthomonas type III effector, is a SUMO (small ubiquitin-like modifier) protease that was reported to bind DNA and to repress defense- and senescence-related plant genes in a process involving its EAR (ERF-associated amphiphilic repression) motifs suggestive of its association with transcription factors (Figure 1, \#3) $[7,11]$. The Shigella type III effector IpaH9.8 was also reported to regulate the activity of the U2AF mRNA splicing factor to downregulate proinflammatory processes (Figure 1, \#4) [12]. Hypermethylation of promoters during Helicobacter pylori infection of gastric epithelial cells has also been involved in the inhibition of transcription and immunosuppression [13]. 


\section{Major recent advances}

Recently, with the growing awareness of the importance of epigenetic regulation, there has been considerable interest in the regulation of host gene expression through chromatin remodeling by bacterial virulence factors [14]. The Shigella type III effector OspF was shown to prevent histone $\mathrm{H} 3$ phosphorylation at serine 10 [15]. This property, associated with the OspF ability to inhibit MAPK activation by virtue of its phosphothreonine lyase activity, would prevent the access of the NF- $\kappa B$ transcriptional activator to specific promoters, such as the interleukin-8 promoter, involved in the mounting of the inflammatory response (Figure 1, \#5) [16]. The suppression of the interferon response by Mycobacterium tuberculosis was also correlated with chromatin remodeling in a process that involves histone deacetylase activity [17]. Bacterial soluble toxins were also shown to modify chromatin, indicating that pathogens can alter gene expression in a paracrine manner in cells that are not directly infected by bacteria. The pore-forming toxins such as the Listeria monocytogenes listeriolysin $\mathrm{O}$, the Clostridium perfringens perfringolysin, and the Streptococcus pneumoniae pneumolysin induce the dephosphorylation of histone $\mathrm{H} 3$ combined with the deacetylation of histone $\mathrm{H} 4$, which correlate with reduced transcription of immunity genes (Figure 1, \#6) [18].

Various pathogenic bacteria such as H. pylori or Bartonella bacilliformis promote cell proliferation. Bacterial toxins can also induce DNA replication and cell proliferation (reviewed in [19]). In addition to their effects on the host cell cytoskeleton, the Escherichia coli cytotoxic necrotizing factor and Bordetella dermonecrotic toxin are deamidases that activate Rho family GTPases, leading to Rhodependent mitogenic signals and to the formation of multinucleated giant cells (Figure 1, \#7). The Pasteurella multocida toxin induces cell proliferation in a process that depends on the heterotrimeric protein Gq/11, but independently of its effects on RhoA (Figure 1, \#8). The stimulatory role of cell proliferation by these toxins during bacterial infection is unclear. In contrast, there is a possible link with cell proliferation induced by the H. pylori CagA type IV effector protein that activates Ras signaling, possibly by directly binding to the growth factor receptor-bound protein 2 (Grb2) adaptor (Figure 1, \#9) [20,21]. CagA, however, has been implicated in multiple functions and could also alter epithelial cell polarity to favor bacterial replication in the gastric mucosa [22].

Bacterial toxins can also induce cell cycle arrest. This particular function could represent a strategy to prevent maturation and exfoliation of epithelial cells to favor bacterial colonization or to alter the integrity of the epithelial barrier. The Clostridium botulinum C3 exoenzyme, an acetyltransferase that inhibits the small GTPase RhoA, induces cell cycle arrest in the $G_{1}$ phase (Figure 1, \#10). The cytolethal distending toxins (CDTs) first reported in E. coli, for which orthologs have been reported in various Gram-negative bacteria (including Shigella dysenteriae, Campylobacter spp., and Salmonella typhi), are tripartite (CdtA, B, and C) holotoxins, with $\mathrm{CdtB}$ being the active component responsible for cell blockade [23]. Although CdtB is an endonuclease, it is unclear whether this activity is directly responsible for DNA damage. CdtB induces the recruitment and activation of Mre11, a nuclease of the central sensor of DNA double-strand breaks, DNA damage, and chromatin injury leading to cell cycle arrest [24] (Figure 1, \#11). Of note, CdtB also harbors a phosphatidyl 3,4,5 inositol phosphatase activity that may also play a role in cell cycle arrest by a mechanism that remains to be clarified [24]. The Cif (cycle inhibiting factor) family of proteins are type III effectors originally reported in enteropathogenic E. coli/enterohemorragic E. coli strains but also found in Yersinia pseudotuberculosis and Burkholderia pseudomallei, which induce cell cycle arrest in a process involving the stabilization of the cyclin-dependent kinase inhibitors $\mathrm{p} 21^{\text {waf1/cip } 1}$ and p $27^{\text {kip }}[25,26]$. In addition to its role in the delivery of type III effectors into host cells, the Shigella protein was shown to bind to Mad2L2, an inhibitor of the anaphase-promoting complex, and thereby to promote cell cycle arrest [27].

\section{Future directions}

How? Many bacterial effectors are proteases/actetyltransferases/deubiquitinases, some of them are endowed with multiple activities, and many of them lack identified targets. The question remains as to what the precise nature and spectrum of these targets are. Addressing these issues could allow us to clarify the links between signaling pathways such as those regulating proteasomal degradation, inflammation, cell proliferation, or cell death.

When? Efforts should also aim at addressing the function of effectors in cell types that play a role during infection. For example, it is conceivable that toxins that induce cell cycle arrest in epithelial cells also act as immunosuppressive agents by preventing the clonal expansion of lymphocytes, as reported for the fusobacterial immunosuppressive protein A (FipA) [28]. The development of new technologies to visualize, in real time, virulence factor delivery into host cells should provide us with clues for understanding how various effectors 'orchestrate' the inflammatory response. 
Why? Besides effects on the global host response, there are, however, examples in which a specific cluster of inflammatory genes is targeted by a given pathogen or by pathogens that specifically alter the host response in a given cell type. Such examples are likely to reflect specific features of the pathogen's physiopathological strategy. Also, in some instances, there are seemingly paradoxical findings for the action of bacterial effectors. For example, a hallmark of bacillary dysentery induced by Shigella is acute inflammation. It has been thought that bacteria need to elicit an inflammatory program to colonize the colonic mucosa. As presented above, this view is now being challenged by the discovery that many Shigella type III effectors appear to downregulate inflammation. This suggests that we are lacking a model that recapitulates critical steps of the infectious process and that efforts need to be made to develop relevant readouts. Recent years have seen the success of what one could call 'molecular mechanistics' of virulence determinants. Along with the molecular dissection of the mode of action of bacterial virulence determinants, getting the right messages from studying genetic reprogramming of host cells by pathogens will obviously imply efforts to integrate the function of these effectors in the infectious context.

\section{Abbreviations \\ CDT, cytolethal distending toxin; Cif, cycle inhibiting factor; CPAF, Clamydia protease activity factor; EAR; ethylene response factor (ERF)-associated amphiphilic repression; Grb2, growth factor receptor-bound protein 2; HIF1 $\alpha$, hypoxia inducible factor 1 alpha; MAPK, mitogen-activated protein kinase; $\mathrm{MHC}$, major histo- compatibility complex; NF- $\kappa \mathrm{B}$, nuclear factor-kappa-B; FipA, fusobacterial immunosuppressive protein A; SUMO, small ubiquitin-like modifier; TLR, Toll-like receptor.}

\section{Competing interests}

The authors declare that they have no competing interests.

\section{Acknowledgments}

The authors are grateful to Philippe Sansonetti for his continuous support and enthusiasm. This work was funded in part by the Institut National de la Santé et de la Recherche Médicale, the Agence Nationale pour la Recherche, and a National Institutes of Health grant (AI067949).

\section{References}

I. Jenner RG, Young RA: Insights into host responses against pathogens from transcriptional profiling. Nat Rev Microbiol 2005, 3:281-94.
2. Lacroix B, Li J, Tzfira T, Citovsky V: Will you let me use your nucleus? How Agrobacterium gets its T-DNA expressed in the host plant cell. Can J Physiol Pharmacol 2006, 84:333-45.

3. Navarro L, Dunoyer P, Jay F, Arnold B, Dharmasiri N, Estelle M, Voinnet $O$, Jones JD: A plant miRNA contributes to antibacterial resistance by repressing auxin signaling. Science 2006, 3 1 2:436-9.

FI000 Factor 8.6 Exceptional

Evaluated by Jiri Friml 04 2006, Andy Maule 08 May 2006, Jennifer Fletcher 08 May 2006, Pierre De Wit 12 May 2006, John W Mansfield 12 May 2006

4. Zhou H, Monack DM, Kayagaki N, Wertz I, Yin J, Wolf B, Dixit VM: Yersinia virulence factor Yopl acts as a deubiquitinase to inhibit NF-kappa B activation. J Exp Med 2005, 202:| 327-32.

FI000 Factor 6.0 Must Read Evaluated by Eric Brown 26 Jan 2006

5. Bliska J: Yersinia inhibits host signaling by acetylating MAP kinases. ACS Chem Biol 2006, 2 I:349-5I.

6. Mukherjee S, Hao Y, Orth K: A newly discovered posttranslational modification: the acetylation of serine and threonine residues. Trends Biochem Sci 2007, 32:210-6.

7. Kay S, Bonas U: How Xanthomonas type III effectors manipulate the host plant cells. Curr Opin Microbiol 2009, I 2:37-43.

8. Zhong $G$, Fan $P$, Ji H, Dong $F$, Huang $Y$ : Identification of a chlamydial protease-like activity factor responsible for the degradation of host transcription factors. J Exp Med 200I, 193:935-42.

FI000 Factor 3.0 Recommended

Evaluated by Richard Stephens 26 Oct 2001

9. Rupp J, Gieffers J, Klinger M, van Zandbergen G, Wrase R, Maass M, Solbach W, Deiwick J, Hellwig-Burgel T: Chlamydia pneumoniae directly interferes with HIF-Ialpha stabilization in human host cells. Cell Microbiol 2007, 9:2181-91.

10. Paschen S, Christian JG, Vier J, Schmidt F, Walch A, Ojcius DM, Häcker G: Cytopathicity of Chlamydia is largely reproduced by expression of a single chlamydial protease. J Cell Biol 2008, 182: | |7-27.

II. Hotson A, Mudgett MB: Cysteine proteases in phytopathogenic bacteria: identification of plant targets and activation of innate immunity. Curr Opin Plant Biol 2004, 7:384-90.

12. Okuda J, Toyotome T, Kataoka N, Ohno M, Abe H, Shimura Y, Seyedarabi A, Pickersgill R, Sasakawa C: The Shigella effector IpaH9.8 binds to a splicing factor U2AF(35) to modulate host immune responses. Biochem Biophys Res Commun 2005, 333:53I-9.

13. Ushijima T, Nakajima T, Maekita T: DNA methylation as a marker for the past and future. J Gastroenterol 2006, 4I:40 I-7.

14. Hamon MA, Cossart P: Histone modifications and chromatine remodeling during bacterial infections. Cell Host Microbe 2008 , 4:100-9.

15. Arbibe L, Kim DW, Batsche E, Pedron T, Mateescu B, Muchardt C, Parsot C, Sansonetti PJ: An injected bacterial effector targets chromatin access for transcription factor NF- kappaB to alter transcription of host genes involved in immune responses. Nat Immunol 2007, 8:47-56.

FI000 Factor 6.4 Must Read Evaluated by Victor Nizet 19 Jan 2007, Craig Roy 19 Apri 2007

16. Li H, Xu H, Zhou Y, Zhang J, Long C, Li S, Chen S, Zhou JM, Shao F: The phosphothreonine lyase activity of a bacterial type III effector family. Science 2007, 3 1 5:1000-3. Erratum in Science 2007. 3 1 7:43-6

17. Wang Y, Curry HM, Zwilling BS, Lafuse WP: Mycobacteria inhibition of IFN-gamma induced HLA-DR gene expression by up-regulating histone deacetylation at the promoter region in human THP-I monocytic cells. J Immunol 2005, I 74:5687-94. 
18. Hamon MA, Batsché E, Régnault B, Tham TN, Seveau S, Muchardt C, Cossart P: Histone modifications induced by a family of bacterial toxins. Proc Natl Acad Sci U S A 2007, 104:13467-72.

FI000 Factor 3.0 Recommended

Evaluated by Victor DiRita 21 Aug 2007

19. Nougayrède JP, Taieb F, De Rycke J, Oswald E: Cyclomodulins: bacterial effectors that modulate the eukaryotic cell cycle. Trends Microbiol 2005, 13:103-10.

20. Mimuro H, Suzuki T, Tanaka J, Asahi M, Haas R, Sasakawa C: Grb2 is a key mediator of Helicobacter pylori CagA protein activities. Mol Cell 2004, 10:745-55.

21. Backert S, Selbach M: Role of type IV secretion in Helicobacter pylori pathogenesis. Cell Microbiol 2008, 10:1573-8I.

22. Tan S, Tompkins L, Amieva M: Helicobacter pylori usurps cell polarity to turn the cell surface into a replicative niche. PLoS Pathogens 2009, 5:el000407.

FI000 Factor 3.0 Recommended

Evaluated by Michael Hensel 2I May 2009

23. Ge Z, Schauer D, Fox J: In vivo virulence properties of bacterial cytolethal-distending toxin. Cell Microbiol 2008, 10:1599-607.
24. Smith J, Bayles D: The contribution of cytolethal distending toxin to bacterial pathogenesis. Crit Rev Microbiol 2006, 32:227-48.

25. Samba-Louaka A, Nougayrède JP, Watrin C, Jubelin G, Oswald E, Taieb F: Bacterial cyclomodulin Cif blocks the host cell cycle by stabilizing the cyclin-dependent kinase inhibitors $\mathrm{p} 2 \mathrm{I}$ and p27. Cell Microbiol 2008, 10:2496-508.

26. Jubelin G, Chavez CV, Taieb F, Banfield MJ, Samba-Louaka A, Nobe R, Nougayrède JP, Zumbihl R, Givaudan A, Escoubas JM, Oswald E: Cycle inhibiting factors (CIFs) are a growing family of functional cyclomodulins present in invertebrate and mammal bacterial pathogens. PLos One 2009, 4:e4855.

27. Iwai H, Kim M, Yoshikawa $Y$, Ashida H, Ogawa M, Fujita Y, Muller D, Kirikae T, Jackson PK, Kotani S, Sasakawa C: A bacterial effector targets Mad2L2, an APC inhibitor, to modulate host cell cycling. Cell 2007, 130:6 II-23.

FI000 Factor 8.0 Exceptional

Evaluated by Berl R Oakley 20 Sep 2007, Pascale Cossart 19 Oct 2007

28. Shenker B, Datar S: Fusobacterium nucleatum inhibits human T-cell activation by arresting cells in the mid-GI phase of the cell cycle. Infect Immun 1995, 63:4830-6. 